\title{
Selectively enhanced expression of prophenoloxidase activating enzyme 1 (PPAE1) at a bacteria clearance site in the white shrimp, Litopenaeus vannamei
}

\author{
In-Kwon Jang, Zhenguo Pang*, Jiaping Yu, Su-Kyoung Kim, Hyung-Cheol Seo and Yeong-Rok Cho
}

\begin{abstract}
Background: The prophenoloxidase-activating (PO activating) system plays an important role in the crustacean innate immunity, particularly in wound healing and pathogen defense. A key member of this system is prophenoloxidase-activating enzyme (PPAE), which is the direct activator of prophenoloxidase (proPO). Despite their importance in crustacean PO activating system, the studies on them remain limited.
\end{abstract}

Results: Here we report on a PPAE of white shrimp, Litopenaeus vannamei (IVPPAE1), which showed 94\% similarity to PPAE1 of Penaeus monodon. We found that IVPPAE1 in fluid hemocytes was down regulated after challenge by Vibrio harveyi but was enhanced when shrimps were exposed to a bacteria-rich environment for long-term. In vivo gene silence of IVPPAE1 by RNAi can significantly reduce the phenoloxidase activity $(\mathrm{PO})$ and increase the susceptibility of shrimps to $V$. harveyi. Although IvPPAE1 was down-regulated in fluid hemocytes by Vibrio challenge, its expression increased significantly in gill after bacteria injection, which is the primary bacteriaclearance tissue.

Conclusion: Suppressed expression in fluid hemocytes and enhanced expression in gill indicates selectively enhanced expression at the bacterial clearance site. This is a novel feature for PPAE expression. The results will contribute to our understanding of the PO activating system in crustaceans.

\section{Background}

Innate immunity is of great importance to insects and crustaceans because they lack antibodies [1]. Innate immunity involves phagocytosis, encapsulation, hemocyte coagulation and activation of the prophenoloxidase (proPO) or melanization cascade [2,3]. Activation of proPO generates phenoloxidase $(\mathrm{PO})$, which catalyzes the oxygenation of monophenols to $o$-diphenols and the oxidation of $o$-diphenols to the corresponding $o$-quinones [4]. These are reactive intermediates for melanin synthesis and other physiological processes such as cuticle sclerotization, wound healing and pathogen sequestration [5].

\footnotetext{
* Correspondence: panishvan@yahoo.com.cn

National Fisheries Research \& Development Institute, \#707, Eulwang dong, Jung-gu, Incheon 400420, Republic of Korea
}

In most cases, the proPO cascade is triggered by a small amount of microbe-derived molecules such as lipopolysaccharides (LPSs), $\beta-1,3$-glucans, or peptidoglycan. Pattern-recognition proteins bind these molecules and initiate the proPO system through a quick proteolytic cascade, and many proteins involved in the proteolytic cascade are serine proteinases [6]. The final serine proteinase that converts the inactive proPO into its active form is called prophenoloxidase-activating enzyme (PPAE) [5].

The proPO genes of insects and crustaceans have been intensively studied [7-14], and models for the serine proteinase cascades regulating proPO activation have been studied in detail in some insects such as Manduca sexta and Tenebrio molitor [15-23]. Studies on proPOactivating cascades are limited in crustaceans. Hitherto, few PPAEs in crustaceans have been reported, except for in crayfish, Pacifastacus leniusculus and shrimp, 
Penaeus monodon [24-26]. In this study, the PPAE1 of white shrimp Litopenaeus vannamei was identified and its expression feature was reported. The new findings of the study will contribute to our understanding of PO activating system of crustaceans.

\section{Results}

\section{Isolation and IvPPAE1 sequence}

The full-length lvPPAE1 cDNA was $1557 \mathrm{bp}$, with an ORF of 1389, a 5'-UTR of $57 \mathrm{bp}$, and a 3'-UTP of 111 bp. The theoretical pI and Mw were 6.96 and $50.4 \mathrm{kDa}$. The proteolytic activation site was found between Arg228 and Ile229. The pI and Mw of activated lvPPAE1 were 4.5 and $25.4 \mathrm{kDa}$. The lvPPAE1 protein showed $94 \%$ identity to pmPPAE1 and $61 \%$ to plPPAE. Three amino acid residues (H270, D319, and S412) corresponded to the catalytic triad of the SP domain and another three amino acid residues (D406, S433, and G435) to the substrate binding sites.

Multiple alignments between the sequences of lvPPAE1, pIPPAE, and pmPPAE1 showed that this gene is relatively well conserved in crustaceans (Figure 1). lvPPAE1 exhibited a typical clip-domain structure, composed of six cysteines at the N-terminus. Similar to other crustacean PPAEs, a glycine-rich domain was found between residues 40 and 130 , with $17.6 \%$ glycines
(16/91, pI = 12.2), and a proline-rich domain was found between residues 194 and 226 , with $39.4 \%$ prolines (13/ 33, $\mathrm{pI}=12.5)$.

\section{IvPPAE phylogenetic analysis}

A phylogenetic tree was constructed using the neighborjoining method (Figure 2) by comparing the deduced amino acid sequence of the conserved SP domain to the 82 clip-domain serine proteinases showing the most similarity or any reported PPAEs. The 83 proteinases were separated into two main branches: one mainly composed of insect prophenoloxidase-activating proteins and the other composed of insect pro-clotting enzymes. The PPAEs of crustaceans were classified into the second main branch and they shared the same and a unique sub-branch. Except for seven pro-clotting enzymes and five trypsins, the biological roles of the clip-domain serine proteinases from the second main branch are still unclear.

Tissue-specific gene expression in healthy shrimp and bacteria-challenged shrimp

lvPPAE1 gene expression was analyzed by qRT-PCR. lvPPAE1 in SPF shrimps was mainly expressed in hemocytes and gill, whilst low level was detected in the other tissues. The relative expression in FHs was about 3.5-

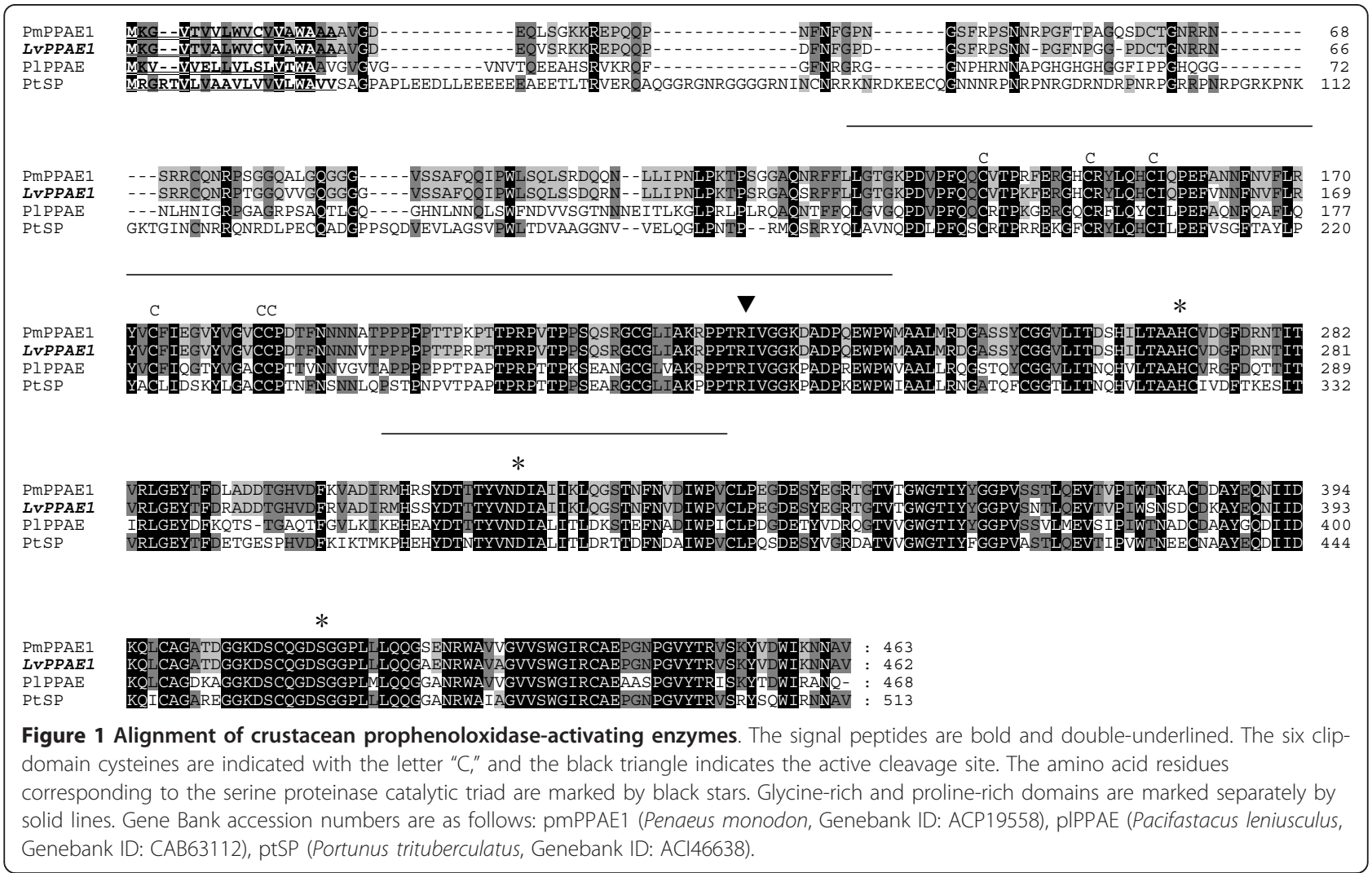




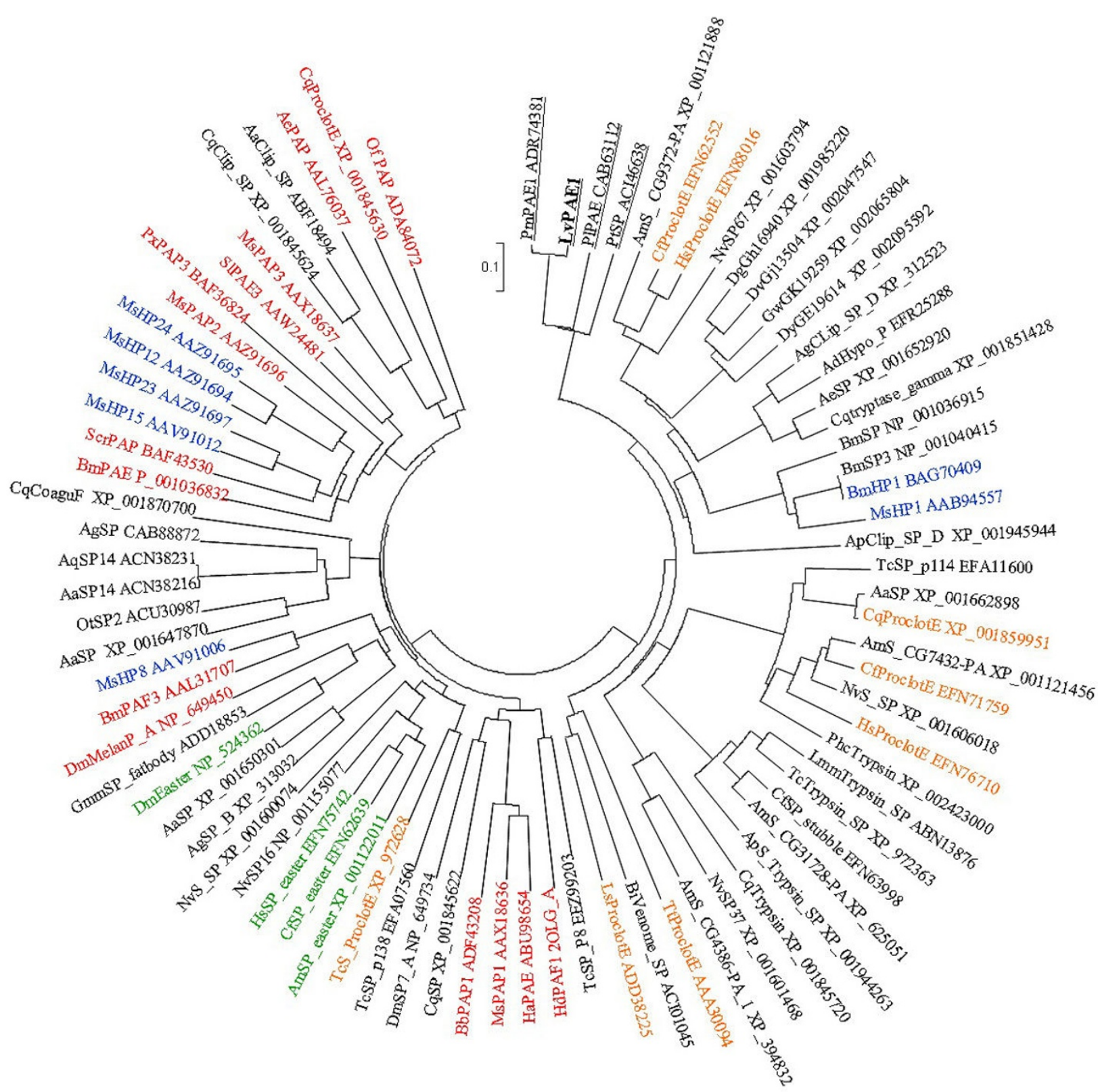

Figure 2 Neighbor-joining phylogenetic tree constructed with the prophenoloxidase-activating enzyme (IvPPAE1) SP domain and the other 82 clip-domain serine proteinases showing the most similarity in prophenoloxidase-activating proteins in insects. Bootstrap sampling was reiterated 1000 times. The scale bar refers to a phylogenetic distance of 0.1 amino acid substitutions per site. Prophenoloxidaseactivating proteins are highlighted in red, and easter-type serine proteases are in green, hemolymph proteases are in blue, and pro-clotting enzymes are in orange. PPAEs in crustaceans are underlined.

fold in gill. Forty eight hours after Vibrio challenge, the relative expression level of lvPPAE1 in gill was as same as that in FHs, which indicated an increase in the gill (Figure 3). Gill samples from the challenged shrimps were checked using microscope with phase contrast mode. Intensive hemocyte aggregation was found in gill canals (Figure 5A, B).

\section{Temporal expression of IVPPAE1 corresponding to bacterial challenge}

Hemocytes and gill were sampled at each time point after the challenge. qRT-PCR showed that the IvPPAE1 mRNA expression level began to decline in FHs in a short time post challenge. This suppression became more obvious after 4 hpc (hour post challenge); the 


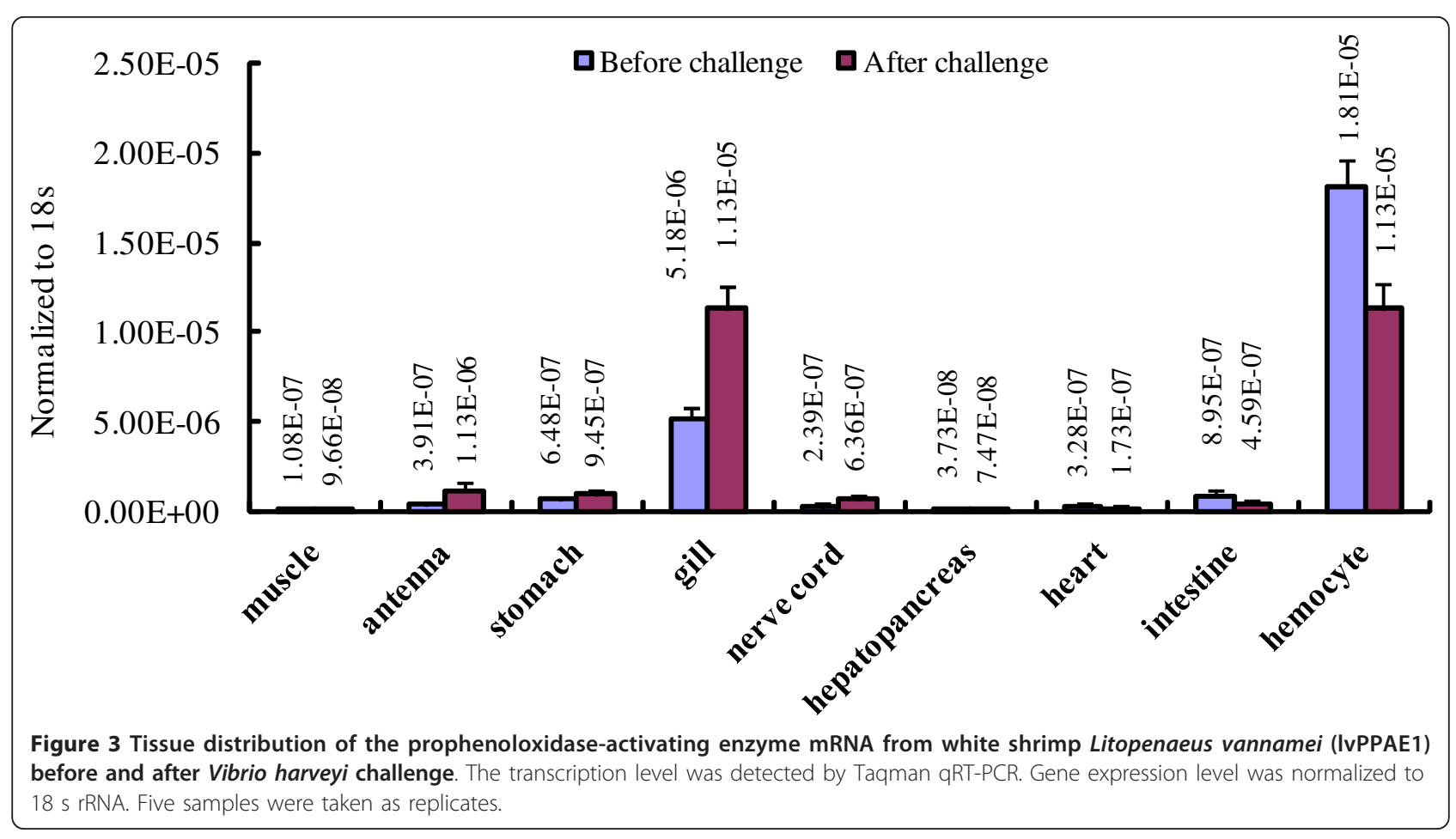

expression level decreased by $60 \%$ compared to the control $(0 \mathrm{~h})$. After $16 \mathrm{hpc}$, the expression recovered slightly but then declined again and remained suppressed after 48 hpc. Multiple comparison between challenged and saline injected groups at each time point showed the suppression of lvPPAE1 were significant at 2, 4, 8 and 24 hpc. Temporal expression in saline injected shrimp was not changed significantly. The two results, normalized to $18 \mathrm{~s}$ and $\beta$-actin separately, were well matched (Figure 4A, B). However, the relative change in expression in gills was different. At $4 \mathrm{hpc}$, lvPPAE1 expression in gill was significantly enhanced to about 2.5 -fold than that of the control $(0 \mathrm{~h})$. After a relative decline at 8 hpc, the expression level increased to 3-fold compared to the control and the high expression was sustained for $48 \mathrm{hpc}$ (Figure 5C).

\section{Gene expression analysis in shrimp reared in different bacterial environments}

Vibrio in the biofloc and filtered seawater was calculated following colony forming unit method after a 24-h culture. The Vibrio number in biofloc seawater was $3.5 \times$ $10^{4} \mathrm{CFU} / \mathrm{ml}$, whereas $45 \mathrm{CFU} / \mathrm{ml}$ were found in filtered seawater (Figure 6A). The relative lvPPAE1 mRNA expression level in $\mathrm{FHs}$ of shrimp reared in different bacterial environments was analyzed by qRT-PCR. Five samples were taken as replicates for expression analysis. The relative lvPPAE1 mRNA expression level in shrimps exposed to a higher bacteria environment was 2.5-fold than that of those exposed to a lower level of bacteria (Figure 6B).

\section{RNA interference of IvPPAE1}

qRT-PCR was used to detect the dsRNA interference effect 12 and $24 \mathrm{~h}$ after the first dsRNA injection and $24 \mathrm{~h}$ after the second injection. Total RNA extracted from the hemocytes of gene-silenced shrimp was analyzed. RNAi strongly inhibited lvPPAE1 expression. The amount of mRNA decreased to less than $5 \%$ of the control $24 \mathrm{~h}$ after the second lvPPAE1 dsRNA injection ( $0 \mathrm{~h}$, Figure 7A).

\section{Susceptibility of shrimp to Vibrio harveyi after gene silencing}

Susceptibility of shrimps to $V$. harveyi was tested after gene silence. About $1 \mathrm{~h}$ after the challenge, shrimps in the gene-silenced group began to show symptoms of slowed mobility and imbalance, as well as mortality after 2 h. Saline-injected shrimp showed symptoms after at least $4 \mathrm{~h}$, and mortality began after $8 \mathrm{~h}$. Mortality in the saline pre-injected group reached about 50\% after $48 \mathrm{~h}$, and all gene-silenced shrimp died within $24 \mathrm{~h}$. No mortality was found in the saline-only injected group and occasional mortality was found in the lvPPAE1 dsRNAonly injected group (Figure 7B).

\section{PO activity assay}

PO activity was analyzed in shrimp injected with genespecific dsRNA, GFP dsRNA, or saline solution. PO- 


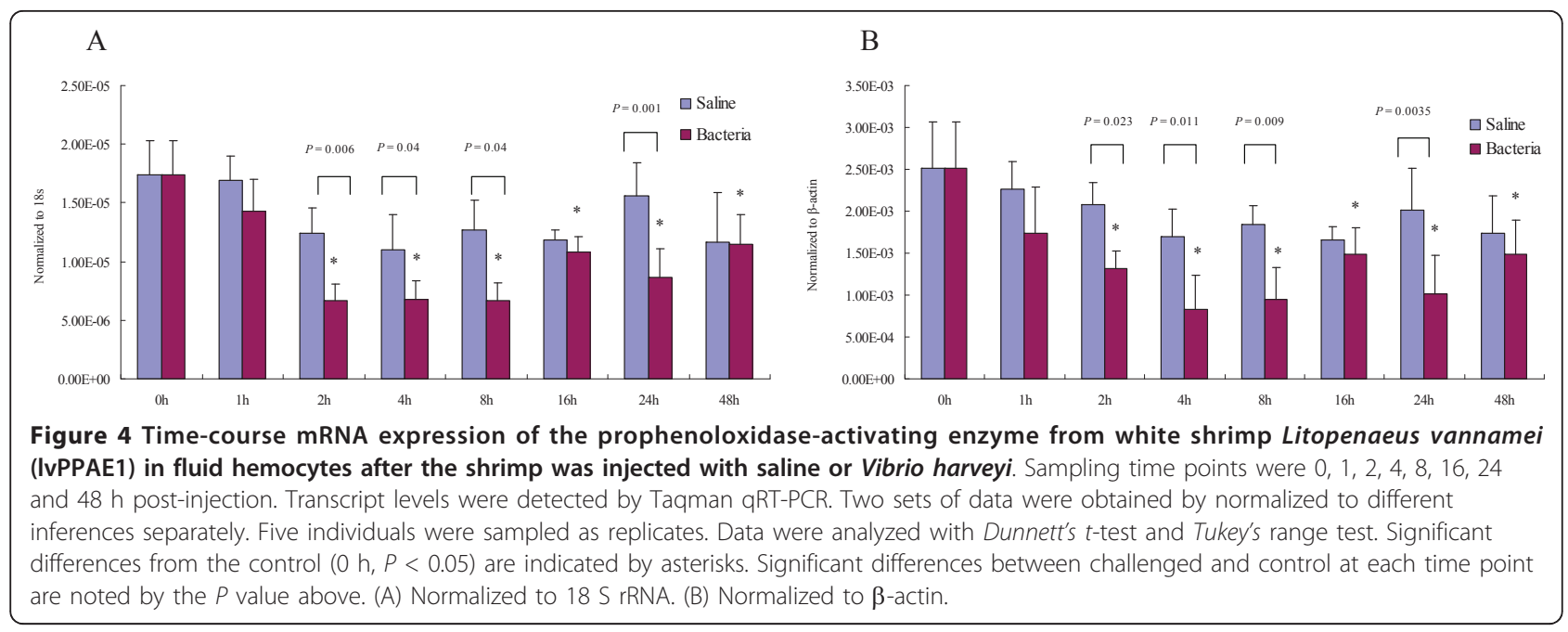

induced activity in the gene-silenced shrimp decreased by approximately $50 \%$ compared to the saline-injected control group. No such decrease was found in the GFP dsRNA-injected shrimp (Figure 7C).

\section{Discussion}

We identified and characterized a clip-domain serine proteinase from the white shrimp, L. vannamei. The deduced amino acid of this gene showed high similarity to that of plPPAE (61\%) and particularly high similarity


Figure 5 Hemocyte aggregation and analysis in gills after Vibrio harveyi challenge. (A) Intensive hemocyte aggregation in gill $4 \mathrm{~h}$ after bacterial challenge. White arrows show aggregated hemocytes in gill canal. (B) Hemocytes released from gill by dissecting. Bar $=20 \mu \mathrm{m}$. (C) Time-course expression of the prophenoloxidase-activating enzyme from white shrimp Litopenaeus vannamei (IVPPAE1) in gill after challenge. Sampling times are 0, 4, 8,16 , and $48 \mathrm{~h}$ post-infection. Five samples were tested as replicates at each time point. Gene expression level was normalized to $\beta$-actin. Data were analyzed with Dunnett's t-test. Significant differences from the control $(0 \mathrm{~h}, P<0.05)$ are indicated with asterisks. to pmPPAE1 (94\%), which is the terminal enzyme in the prophenoloxidase-activating cascade $[25,26]$. Thus, we named this gene lvPPAE1. Multiple alignment results indicated that this gene is rather well conserved in crustaceans. Interestingly, phylogenetic analyses revealed that all PPAEs in crustaceans branched off into the cluster of insect pro-clotting proteinases rather than insect PPAEs. Besides with molecular structure deviation, PPAEs in crustaceans can proteolyses proPO dependently [27], unlike most of insect PPAEs that need cofactors to active proPOs [6]. Another major difference between PPAEs in crustaceans and insects is the different regulation type to pathogen invasion. Three prophenoloxidase activating proteinase of the horn worm Manduca sexta were reported to be stimulated or upregulated by injection of Micrococcus lysodeikticus or Escherichia coli $[17,28,29]$. Five putative PPAEs of the fly Drosophila were induced to higher levels after E. coli and Micrococcus luteus infection [30]. Two potential PPAEs of the mosquito Anopheles gambiae were up-

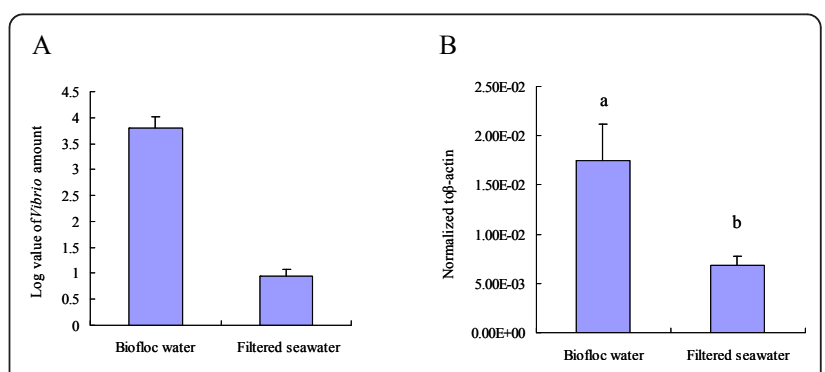

Figure 6 Vibrio amounts in different water conditions and corresponding IvPPAE1 mRNA expression levels. Five samples were taken as replicates. (A) Log value of the Vibrio colony amount per milliliter biofloc water and filtered seawater. (B) qRT-PCR analysis of IVPPAE1 mRNA expression level in FHs of shrimp reared in biofloc or filtered seawater. Data were analyzed with the unpaired $t$-test. Different letters indicate significantly different means $(P<0.05)$. 


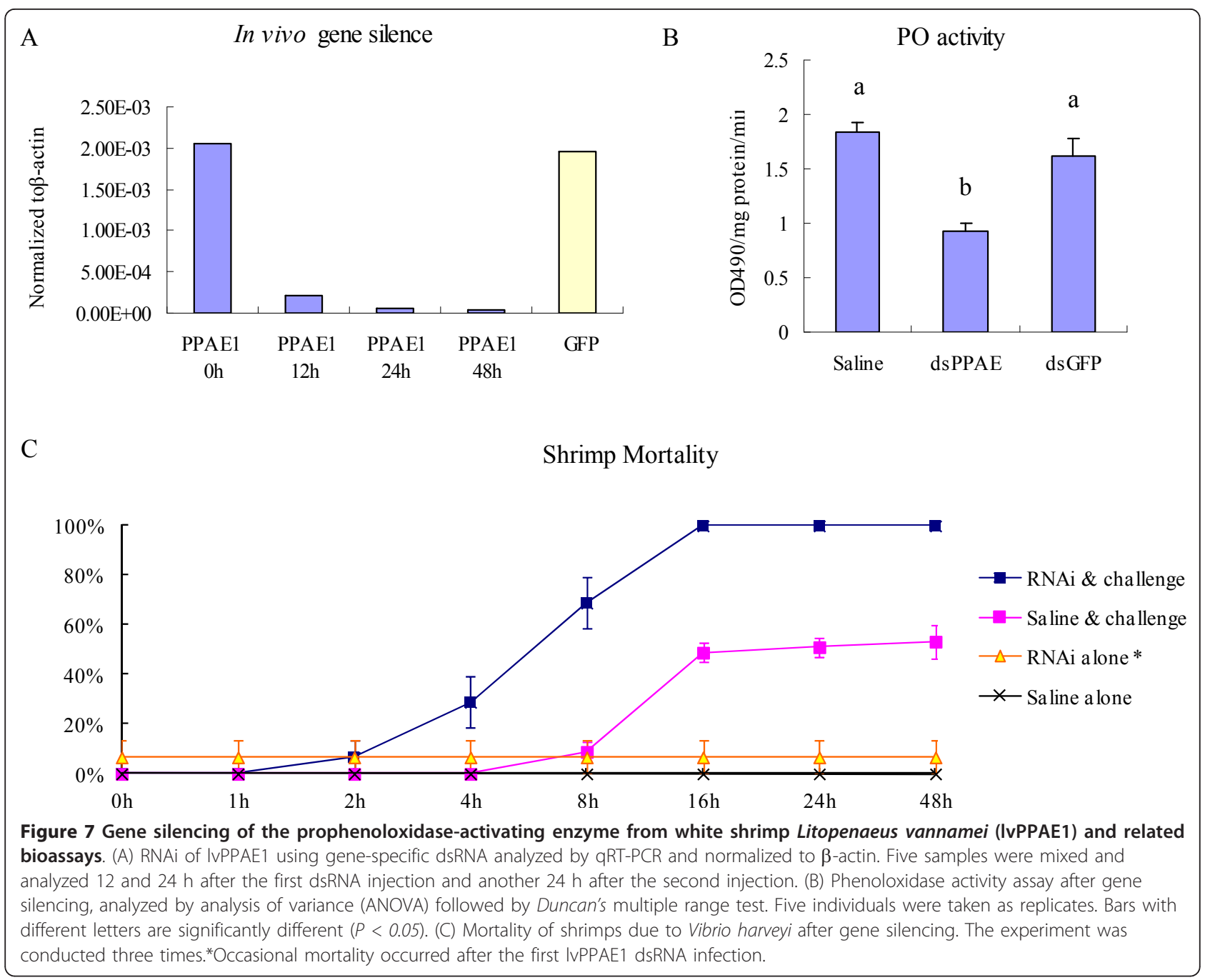

regulated by bacteria E. coli or M. luteus and parasite Plasmodium berghei [31]. A prophenoloxidase activation proteinase of the silk worm Samia cynthia ricini was inducible by cell wall components of gram-positive bacteria [32]. Contrary to those insect PPAEs, The crayfish PPAE transcript level was reported to be unaffected upon Aeromonas hydrophila challenge [33]. The pmPPAE1 transcript level in P. monodon was down regulated 3-24 h after Vibrio injection [25]. In the present study, the lvPPAE1 transcript abundance was reduced within $48 \mathrm{~h}$ after Vibrio challenge. All reported crustacean PPAEs as well as our study showed their transcript levels were either not affected or down-regulated within $48 \mathrm{~h}$, contrary to PPAEs in insects that were up-regulated or stimulated within $24 \mathrm{~h}$ after challenge. Dissimilarity in proteinase structures and functional features imply certain degree of difference exists between crustaceans and insects for the prophenoloxidase-activating mechanism, particularly in the down stream.
It has been reported that the PPAE1 mRNA of crustaceans were found specially in hemocyte and free from other tissues [25]. Our tissue distribution analysis by qRT-PCR also revealed that lvPPAE1 transcript level was very low in muscle, antenna gland, nerve cord and digesting system, and this minimum expression also could be caused by a few amount of hemocytes penetrated into the tissues [5]. The most convincing proof was that the lvPPAE1 transcript level in those tissues didn't change after bacteria challenge, suggesting that these tissues were in fact free of lvPPAE1 transcripts. This conclusion is in accordance with the previous report [25]. However, it is notable that during bacteria clearance process in shrimps, bacteria or foreign materials would be encapsulated by hemocytes to form hemocyte-foreign material aggregation and this kind of aggregation happens mainly in gill, which is the main bacteria or alien materials clearance site [34]. This phenomenon was also confirmed by our microscope observation (Figure 5A, B). In another word, gill is closely 
related with hemocytic immunity in host defense of shrimps. By sensitive qRT-PCR method, we successfully found out the inevitable repercussion of this close relation: The lvPPAE1 mRNA transcripts represented a considerable level and even higher after Vibrio challenge.

Our RNAi experiment showed silence of lvPPAE1 significantly reduced the PO activity and increased the susceptibility to $V$. harveyi. qRT-PCR revealed shrimps exposed in a bacteria rich environment for a long-term presented a higher lvPPAE1 transcript level. Those results suggested the importance of lvPPAE1 in the host defense of L. vannamei. It is thus odd to find the expression of lvPPAE1 was depressed when shrimps tried to fight against the injected bacteria. Same phenomenon was found in another shrimp species $P$. mono$d o n$, yet the author didn't give an explanation. Our findings in gill gave a clue to answer the doubtful question. Although the transcript expression of lvPPAE1 in gill was in fact due to the fixed hemocytes $(\mathrm{GH})$, the transcript level of GHs showed different regulation type than in FHs. Contrary to the continuous dropping trend in FHs, the transcript level of lvPPAE1 in gill was stimulated quickly and arrived to a peak at $4 \mathrm{~h}$ when the depression in FHs was at the upmost degree. Higher lvPPAE1 mRNA transcript level was maintained in gill within $48 \mathrm{~h}$ when the lvPPAE1 was continuously depressed in FHs. By now, a new conclusion was drawn that the lvPPAE1 was not simply down-regulated by Vibrio challenge but also up-regulated in bacteria clearance site. By the current data, it can not be asserted that the overall lvPPAE1 transcript level was depressed or enhanced shortly after the Vibrio challenge; we thus call the expression feature as the "selective enhanced expression". Because the selective enhanced expression in gill happens simultaneously with the suppression in fluid hemocytes, the endogenous regulation mechanism for the short time enhanced expression in bacteria clearance site is probably different from the enhanced expression in FHs under a long-term, bacteria-rich environment stress. Our current study on lvPPAE1 indicated that the endogenous regulation mechanism for shrimp immunity is far from clarified.

\section{Conclusion}

A prophenoloxidase activating enzyme (lvPPAE1) of the white shrimp L. vannamei was cloned and sequenced. In vivo gene silence of lvPPAE1 significantly deduced the PO activity and increased the susceptibility of shrimps to gram negative bacteria $V$. harveyi. The overall tissue distribution analysis showed the lvPPAE1 was mainly expressed in fluid hemocytes $(\mathrm{FH})$ and gill due to the aggregated hemocytes in canal $(\mathrm{GH})$. The lvPPAE1 expression was significantly enhanced in gill within $2 \mathrm{~h}$ after $V$. harveyi challenge. In the case of $\mathrm{FH}$,
lvPPAE1 expression was suppressed within $48 \mathrm{~h}$ after a single challenge, whereas higher expression was found when shrimp was exposed to a high bacterial environment over the long term. The different gene expression responses to bacterial challenge in $\mathrm{FH}$ and gill suggest enhanced lvPPAE1 expression at the bacteria-clearance site.

In conclusion, the lvPPAE1 is involved in melanization cascade and plays an important role in shrimp host defense against bacteria. The expression in FH was suppressed in a short term after $V$. harveyi challenge but was up regulated under bacterial press for a long term. The lvPPAE1 represents a feature of selective enhanced expression in bacteria clearance site after challenge. This is a novel characteristic for crustacean PPAEs expression.

\section{Methods}

Animals, sample preparation and RNA preparation

Specific pathogen-free (SPF) L. vannamei (10 g $\pm 1.2 \mathrm{~g}$ mean \pm SD) were obtained from a biofloc water culture system at the NFRDI (Seoul, Korea) and were maintained in filtered running seawater for two weeks, if needed. All tissue preparations were performed as bellow: Tissues such as muscle, stomach, antenna gland, nerve cord, intestine, hepatopancreas, or gill were obtained by dissection. Hemolymph was collected from the ventral hemolymph sinus of L. vannamei with a 3 $\mathrm{ml}$ RNase-free syringe containing on ice pre-cooled anticoagulant (113 mM glucose, $27.2 \mathrm{mM}$ sodium citrate, $2.8 \mathrm{mM}$ citric acid, and $71.9 \mathrm{mM} \mathrm{NaCl}$ ). The hemocyte cell pellet was collected by centrifugation at $700 \times \mathrm{g}$ and $4^{\circ} \mathrm{C}$ for $10 \mathrm{~min}$ followed by two rinses with anticoagulant. All tissues were placed immediately in $200 \mu \mathrm{l}$ RNA later reagents after preparation (Ambion, Austin, TX, USA). Total RNA was extracted with the RNeasy Mini Kit (Qiagen, Valencia, CA, USA) and further purified with DNase I (Qiagen), according to the manufacturer's protocol.

\section{cDNA synthesis and rapid amplification of cDNA ends}

The first-strand cDNA for 5' rapid amplification of cDNA ends (RACE) and 3' RACE was synthesized separately with the SMARTer ${ }^{\mathrm{TM}}$ RACE cDNA Amplification Kit (Clontech, Mountain View, CA, USA), according to the user's manual. The first-strand cDNA was diluted 20 times to serve as the RACE template. A degenerate of the gene-specific primer lvPPAERACE3' was designed based on other crustacean PPAE sequences $[25,26]$. PCR amplification was performed with the specific primer and two universal primer provided by the kit. The touchdown PCR conditions were as follows: five cycles of $94^{\circ} \mathrm{C}$ for $30 \mathrm{~s}$ and $72^{\circ} \mathrm{C}$ for $3 \mathrm{~min}$; five cycles of $94^{\circ} \mathrm{C}$ for $30 \mathrm{~s}, 70^{\circ} \mathrm{C}$ for $30 \mathrm{~s}$, and $72^{\circ} \mathrm{C}$ for $3 \mathrm{~min} ; 25$ cycles of 
$94^{\circ} \mathrm{C}$ for $30 \mathrm{~s}, 68^{\circ} \mathrm{C}$ for $30 \mathrm{~s}$, and $72^{\circ} \mathrm{C}$ for $3 \mathrm{~min}$. PCR products were then subcloned into the TOPO vector (Invitrogen, Carlsbad, CA, USA) and sequenced. The fragment sequence was confirmed by the BLAST program on NCBI by comparison with other shrimp serine proteases. A new primer, lvPPAERACE5', was designed based on the partial sequence of lvPPAE1. 5' RACE was performed with the new primer and universal primers mentioned above with the same PCR conditions as 3' RACE. Then the 5' RACE product was subcloned into the TOPO vector and sequenced. After the 5' RACE sequence was confirmed by BLAST, 3' RACE fragment and 5' RACE fragment sequences were joined using the Mega 4 program [35].

\section{Sequence analysis}

The open reading frame (ORF) of the gene was analyzed by the ORF finder program on NCBI. The nucleotide sequence and deduced amino acid sequences were analyzed by the BLAST program on NCBI. Multiple alignments were performed with the Clustal W2 program http://www.ebi.ac.uk/Tools/clustalw2/, and the result was viewed with Genedoc 2.7.0 [36]. A neighbor-joining phylogenetic tree was constructed based on the SP domain of the deduced amino acid sequences of lvPPAE1 and the 82 clip-domain serine proteinases showing highest similarity or the prophenoloxidase-activating proteins of insects using the Mega 4 program [35]. The lvPPAE1 signal peptide was predicted with the SignalP 3.0 server http://www.cbs.dtu.dk/services/SignalP/. The theoretical isoelectric point (pI) and molecular weight $(\mathrm{Mw})$ of the proteins were estimated online http://isoelectric.ovh.org/.

\section{Quantitative real-time PCR for mRNA detection}

A Taqman probe-based quantitative reverse transcription PCR (qRT-PCR) technique was taken to test the lvPPAE1 transcript level. Tissue distribution or mRNA expression under different circumstance stresses can thus be evaluated by this method. A pair of gene-specific primers qRTPPAEf, qRTPPAEr and a Taqman qRTPPAEp probe were designed from the full-length lvPPAE1 cDNA sequence using PrimerExpress software (Applied Biosystems Pty Ltd., Melbourne, Australia). One-step qRT-PCR was accomplished with the One Step PrimeScript ${ }^{\mathrm{TM}}$ RT-PCR perfect real time Kit (Takara Bio). The reaction mixture consisted of $10 \mu \mathrm{l}$ $2 \times$ One-Step RT-PCR Buffer III, 2 units of Takara Ex Hot Start Taq enzyme, $0.4 \mu$ reverse transcript enzyme Mix II, and $0.4 \mu \mathrm{M}$ each of forward primer, reverse primer, and Taqman probe in a final reaction volume of 20 $\mu$ l. The PCR conditions were as follows: $42^{\circ} \mathrm{C}$ for $5 \mathrm{~min}$, $95^{\circ} \mathrm{C}$ for $10 \mathrm{~s}$, followed by 40 cycles of $95^{\circ} \mathrm{C}$ for $5 \mathrm{~s}$ and $60^{\circ} \mathrm{C}$ for $30 \mathrm{~s}$. Fluorescent signal detection was started from the first cycle of the annealing stage. The $18 \mathrm{~s}$ ribosomal RNA (18 s) and $\beta$-actin was taken as references with primers and probe shown in Table 1.

\section{Vibrio challenge for temporal expression assay}

$V$. harveyi KCCM 40866 obtained from the Korean Culture Center of Microorganism (KCCM) was used in a preliminary challenge trial to determine its pathogenicity. Then it was further selectively enriched from the hepatopancreas of freshly killed shrimp in the preliminary challenge on tryptic soy agar (Difco, Franklin Lakes, NJ, USA) plate. Harvested bacteria were diluted with physiological saline $(154 \mathrm{mM})$ to an $\mathrm{OD}_{540}$ of $0.09\left(10^{6}\right.$ CFU). The diluted bacteria $(100 \mu \mathrm{l})$ were injected into the third abdominal segment. SPF shrimps (about 10 gram) from biofloc culture system were raised in filtered sea water for 2 weeks before challenge. One hundred and twenty individuals were challenged and a control group of 50 individuals were injected with the same volume of saline. Gill and hemocyte were sampled at 0 h, 1 h, 2 h, $4 \mathrm{~h}, 8 \mathrm{~h}, 12 \mathrm{~h}$ and $48 \mathrm{~h}$ after bacteria or saline injection. Five shrimps for each group were sampled as replicates at each time point separately. Temporal expression was detected by qRT-PCR and the relative expression data were analyzed by Dunnett's $t$ - test and Tukey's range test.

\section{Vibrio count in biofloc and filtered sea water and corresponding IvPPAE1 mRNA expression}

Water samples $(200 \mu \mathrm{l})$ from biofloc water or filtered sea water were spread on $0.2 \%$ sodium-chloride thiosulfate citrate bile salt agar (Difco) and incubated at $28^{\circ} \mathrm{C}$ for $24 \mathrm{~h}$ before the colonies were counted. Vibrio colony numbers were counted three times everyday continuously for 4 days. Fluid hemocytes were extracted from SPF shrimps (about 10 gram) raised in biofloc water culture system or filtered running seawater and the lvPPAE1 mRNA level were measured and compared with qRT-PCR. Five shrimps were sampled as replicates from the two culture systems separately. The deference of PO activity was analyzed by unpaired $t$-test for unequal variance.

\section{dsRNA production}

First-strand cDNA was synthesized with the PrimeScript ${ }^{\mathrm{TM}}$ First-Strand cDNA Synthesis Kit (Takara Bio) with the oligo dT Primer, according to the manufacturer's protocol. A pair of forward and reverse primers with a T7 promoter oligo anchor (dslvPPAEf, dslvPPAEr) amplifying a fragment of 542 lvPPAE1 nuclei fragments was designed. The PCR amplification was conducted with a reaction mixture of $2 \mu \mathrm{l} 10 \times$ PCR buffer, $1.6 \mu \mathrm{l}$ dNTP mixture, and $0.1 \mu \mathrm{l}$ ExTaq enzyme with a final primer concentration of $0.4 \mu \mathrm{M}$. One $\mu \mathrm{l}$ 
Table 1 Primers and probes used in the experiments

\begin{tabular}{|c|c|c|}
\hline Name & Nucleotide sequence & Note \\
\hline dsEGFPf & 5'-GAATTAATACGACTCACTATAGGGAGACGTGACCACCCTGACCTA-3' & RNAi control forward primer \\
\hline dsEGFPr & 5'-GAATTAATACGACTCACTATAGGGAGAATGCCGTTCTTCTGCTTG-3' & RNAi control reverse primer \\
\hline IvPPAERACE3' & 5'-AGTCACATYCTYACKGCYGCSCACTG-3' & 3' RACE primer \\
\hline IVPPAERACE5' & 5'-GGTCGAAGCCGTCAACGCAGTGC-3' & 5' RACE primer \\
\hline dslvPPAEf & 5'-GAATTAATACGACTCACTATAGGGAGACTTCCGTCCTTCCAACAAT-3' & dsRNA production forward primer \\
\hline dslvPPAEr & 5'-GAATTAATACGACTCACTATAGGGAGAGCCTCTTGGCGATCAGTC-3' & dsRNA production forward primer \\
\hline qRTPPAEf & 5'-AGTTCCTACGACACGACCACCTA-3' & qRT-PCR PPAE1 forward primer \\
\hline qRTPPAEr & 5'-TCGACGTTGAAGTTGGTGCTT-3' & qRT-PCR PPAE1 reverse primer \\
\hline qRTPPAEP & 5'-AACGACATCGCCATCATCAAGCTGC-3' & qRT-PCR probe \\
\hline qRTBf & 5'-CGAGGTATCCTCACCCTGAAAT-3' & qRT-PCR $\beta$-actin forward primer \\
\hline qRT $\beta r$ & 5'-GTGATGCCAGATCTTCTCCATGT-3' & qRT-PCR $\beta$-actin reverse primer \\
\hline$q R T \beta p$ & 5'-CGAGCACGGCATCGTCACCAA-3' & qRT-PCR $\beta$-actin probe \\
\hline qRT18sf & 5'-TGCTCAGAGCAGGCTGGTTT-3' & qRT-PCR 18s forward primer \\
\hline qRT18sr & 5'-GAGGTCCTGTTCCAATCATTCCA-3' & qRT-PCR $18 \mathrm{~s}$ reverse primer \\
\hline qRT18sp & 5'-TGCTTACAGCCCGAATGGTCGTGC-3' & qRT-PCR 18s probe \\
\hline
\end{tabular}

first-strand cDNA was added to make a final volume of $20 \mu \mathrm{l}$ with PCR water. The PCR conditions were as follows: 35 cycles of $94^{\circ} \mathrm{C}$ for $30 \mathrm{~s}, 59^{\circ} \mathrm{C}$ for $30 \mathrm{~s}$, and $72^{\circ} \mathrm{C}$ for $1 \mathrm{~min} 30 \mathrm{~s}$. PCR fragments were purified by agarose gel electrophoresis and subcloned into the TOPO vector (Invitrogen). Sequences were confirmed by sequence alignment and then used as the template for a secondary PCR with T7 promoter primers only. The secondary PCR product was then used as a template to produce dsRNA. dsRNA was produced and purified using the RiboMAX ${ }^{\mathrm{TM}}$ T7 Express System (Promega, Madison, WI, USA), according to the user's manual. dsRNA concentration was calculated by measuring the absorbance at $260 \mathrm{~nm}$ with a spectrophotometer (Cary 100 scan spectrophotometer, Varian). An alien GFP protein sequence was amplified with the PEGFP-1 (Clontech) reconstructed vector, using the dsGFPf forward primer and the dsGFPr reverse primer (encoding 303 nuclei), and the produced dsRNA fragment was used as a control.

\section{In vivo gene silencing}

lvPPAE1 and GFP protein dsRNA were diluted in $0.9 \%$ saline separately. Diluted dsRNA was injected into the third abdominal segment using a $1 \mathrm{ml}$ sterile hypodermic syringe with a 26 gauge needle. Approximately $1 \mu \mathrm{g}$ lvPPAE1 or GFP dsRNA was used per $1 \mathrm{~g}$ shrimp. Another injection with the same amount of dsRNA was performed $24 \mathrm{~h}$ later. The gene silencing effect was tested by qRT-PCR.

\section{Analysis of susceptibility to $V$. harveyi}

Susceptibility of lvPPAE1 gene silenced or control shrimp to $V$. harveyi was evaluated. SPF shrimps from biofloc system were pre-raised in $26^{\circ} \mathrm{C}$ filtered sea water for two weeks and used for experiment. Shrimps were challenged simultaneously with the second dsRNA injection for gene silencing. Mortality of gene silenced or control shrimp was monitored within $48 \mathrm{~h}$. Two additional groups injected with only saline or only lvPPAE1 dsRNA were set as controls to determine mortality from the procedure. Thirty individuals for each group were used to calculate the mortality rate and the experiment were repeated for three times.

\section{Phenoloxidase activity assay}

Hemolymph of shrimps from the gene silenced or control group were extracted with anticoagulant buffer, centrifuged at $700 \times \mathrm{g}$ and $4^{\circ} \mathrm{C}$ for $15 \mathrm{~min}$. The cell pellet was rinsed twice with anticoagulant buffer, resuspended, and centrifuged at $16000 \times \mathrm{g}$ and $4^{\circ} \mathrm{C}$ for $5 \mathrm{~min}$. An aliquot $(500 \mu \mathrm{l})$ of ice-cold homogenizer buffer containing $10 \mathrm{mM}$ sodium cacodylate, $5 \mathrm{mM} \mathrm{CaCl}_{2}$, pH 7.0 (Sigma, Munich, Germany) was added, and the cell pellet was homogenized for $2 \mathrm{~min}$ at full speed to release the proteins. The cell homogenate was then centrifuged at $10000 \times$ g and $4^{\circ} \mathrm{C}$ for $10 \mathrm{~min}$, and the supernatant (LPS) was used for the PO activity test. LPS $(100 \mu \mathrm{l})$ and $50 \mu \mathrm{l}$ laminarin $(0.1 \mathrm{mg} /$ $\mathrm{ml}$, Sigma) was mixed in a 96-well ELISA plate and incubated at $27^{\circ} \mathrm{C}$ for $10 \mathrm{~min}$. L-3, 4-dihydroxyphenylalanine ( $50 \mu \mathrm{l}, 3 \mathrm{mg} / \mathrm{ml}$ ) was added, and PO activity was measured with an ELISA reader, using dynamic read style, for 20 min at room temperature. Absorbance at $490 \mathrm{~nm}$ was measured from 10 to $20 \mathrm{~min}$. Protein concentration was measured with a BCA ${ }^{\mathrm{TM}}$ Protein Assay Kit (Pierce, Rockford, IL, USA). PO activity was calculated as OD490/mg LPS protein/min. Data was analyzed by analysis of variance followed with Duncan's multiple range test. Five individuals were sampled as replicates. 


\section{Acknowledgements}

This work was supported by the Project "Environmentally-friendly BFT shrimp culture technology" (No. RP2011-AQ-122), National Fisheries Research \& Development Institute (NFRDI), Republic of Korea.

\section{Authors' contributions}

IKJ designed the study and gave important comments to the MS. ZGP performed the statistical analysis and wrote the MS. ZGP and JPY carried out sequencing, sequence alignment, qRT-PCR analysis, RNAi and challenge experiment and PO assay. SKK participated in PO assay and Vibrio counting HCS and YRC prepared materials and the field facility. They also donated animals for the study.

Received: 1 August 2011 Accepted: 30 December 2011

Published: 30 December 2011

\section{References}

1. Iwanaga Si, Bok LL: Recent advances in the innate immunity of invertebrate animals. Journal of Biochemistry and Molecular Biology 2005, 38:128-150.

2. Jiravanichpaisal $P$, Lee BL, Söderhäll K: Cell-mediated immunity in arthropods: Hematopoiesis, coagulation, melanization and opsonization. Immunobiology 2006, 211:213-236.

3. Lemaitre $B$, Hoffmann J: The host defense of Drosophila melanogaster. Annual Review of Immunology 2007, 25:697-743.

4. Cerenius L, Lee BL, Söderhall K: The proPO-system: pros and cons for its role in invertebrate immunity. Trends in Immunology 2008, 29:263-271.

5. Cerenius $L$, Söderhäll $K$ : The prophenoloxidase-activating system in invertebrates. Immunological Reviews 2004, 198:116-126.

6. Cerenius L, Kawabata SI, Lee BL, Nonaka M, Söderhäll K: Proteolytic cascades and their involvement in invertebrate immunity. Trends in Biochemical Sciences 2010, 35:575-583.

7. Ai HS, Liao JX, Huang XD, Yin ZX, Weng SP, Zhao ZY, Li SD, Yu XQ, He JG: A novel prophenoloxidase 2 exists in shrimp hemocytes. Developmental \& Comparative Immunology 2009, 33:59-68.

8. Abdel-latief M, Hilker M: Innate immunity: Eggs of Manduca sexta are able to respond to parasitism by Trichogramma evanescens. Insect Biochemistry and Molecular Biology 2008, 38:136-145.

9. Ai HS, Huang YC, Li SD, Weng SP, Yu XQ, He JG: Characterization of a prophenoloxidase from hemocytes of the shrimp Litopenaeus vannamei that is down-regulated by white spot syndrome virus. Fish \& Shellfish Immunology 2008, 25:28-39.

10. Asano T, Ashida M: Cuticular pro-phenoloxidase of the silkworm, Bombyx mori. Purification and demonstration of its transport from hemolymph. $J$ Biol Chem 2001, 276:11100-12.

11. Chen P, Li J, Li J, Liu P, Gao B, Wang Q: Molecular cloning and characterization of prophenoloxidase gene in swimming crab Portunus trituberculatus. Fish \& Shellfish Immunology 2010, 28:106-112.

12. Fujimoto K, Masuda K, Asada N, Ohnishi E: Purification and characterization of prophenoloxidases from pupae of Drosophila melanogaster. J Biochem 1993, 113:285-91.

13. Kawabata T, Yasuhara Y, Ochiai M, Matsuura M, Ashida M: Molecular cloning of insect pro-phenol oxidase: a copper-containing protein homologous to arthropod hemocyanin. Proc Natl Acad Sci USA 1995, 92:7774-8

14. Sritunyalucksana K, Cerenius L, Söderhall K: Molecular cloning and characterization of prophenoloxidase in the black tiger shrimp, Penaeus monodon. Developmental \& Comparative Immunology 1999, 23:179-186.

15. Gupta S, Wang Y, Jiang H: Purification and characterization of Manduca sexta prophenoloxidase-activating proteinase-1, an enzyme involved in insect immune responses. Protein Expression and Purification 2005, 39:261-268.

16. Ji C, Wang Y, Ross J, Jiang H: Expression and in vitro activation of Manduca sexta prophenoloxidase-activating proteinase-2 precursor (proPAP-2) from baculovirus-infected insect cells. Protein Expression and Purification 2003, 29:235-243.

17. Jiang $H$, Wang $Y, Y u X Q$, Zhu $Y$, Kanost M: Prophenoloxidase-activating proteinase-3 (PAP-3) from Manduca sexta hemolymph: a clip-domain serine proteinase regulated by serpin-1J and serine proteinase homologs. Insect Biochemistry and Molecular Biology 2003, 33:1049-1060.
18. Lee KY, Zhang R, Kim MS, Park JW, Park HY, Kawabata SI, Lee BL: A zymogen form of masquerade-like serine proteinase homologue is cleaved during pro-phenoloxidase activation by $\mathrm{Ca}^{2+}$ in coleopteran and Tenebrio molitor larvae. European Journal of Biochemistry 2002, 269:4375-4383.

19. Wang $Y$, Jiang $H$ : Purification and characterization of Manduca sexta serpin-6: a serine proteinase inhibitor that selectively inhibits prophenoloxidase-activating proteinase-3. Insect Biochemistry and Molecular Biology 2004, 34:387-395.

20. Wang $Y$, Jiang $H$ : Reconstitution of a branch of the Manduca sexta prophenoloxidase activation cascade in vitro: Snake-like hemolymph proteinase 21 (HP21) cleaved by HP14 activates prophenoloxidaseactivating proteinase-2 precursor. Insect Biochemistry and Molecular Biology 2007, 37:1015-1025.

21. Wang $Y$, Zou $Z$, Jiang $H$ : An expansion of the dual clip-domain serine proteinase family in Manduca sexta: Gene organization, expression, and evolution of prophenoloxidase-activating proteinase-2, hemolymph proteinase 12, and other related proteinases. Genomics 2006, 87:399-409.

22. Zhang R, Cho HY, Kim HS, Ma YG, Osaki T, Kawabata SI, Söderhall K, Lee BL: Characterization and properties of a 1,3- $\beta$-d-glucan pattern recognition protein of Tenebrio molitor larvae that is specifically degraded by serine protease during prophenoloxidase activation. Journal of Biological Chemistry 2003, 278:42072-42079.

23. Zou Z, Wang Y, Jiang H: Manduca sexta prophenoloxidase activating proteinase-1 (PAP-1) gene: Organization, expression, and regulation by immune and hormonal signals. Insect Biochemistry and Molecular Biology 2005, 35:627-636.

24. Charoensapsri W, Amparyup P, Hirono I, Aoki T, Tassanakajon A: PmPPAE2, a new class of crustacean prophenoloxidase (proPO)-activating enzyme and its role in PO activation. Developmental \& Comparative Immunology 2011, 35:115-124.

25. Charoensapsri W, Amparyup P, Hirono I, Aoki T, Tassanakajon A: Gene silencing of a prophenoloxidase activating enzyme in the shrimp, Penaeus monodon, increases susceptibility to Vibrio harveyi infection. Developmental \& Comparative Immunology 2009, 33:811-820

26. Wang R, Lee SY, Cerenius L, Söderhäll K: Properties of the prophenoloxidase activating enzyme of the freshwater crayfish, Pacifastacus leniusculus. European Journal of Biochemistry 2001, 268:895-902.

27. Aspán A, Huang TS, Cerenius L, Söderhäll K: cDNA cloning of prophenoloxidase from the freshwater crayfish Pacifastacus leniusculus and its activation. Proceedings of the National Academy of Sciences of the United States of America 1995, 92:939-943.

28. Jiang $H$, Wang $Y$, Kanost MR: Pro-phenol oxidase activating proteinase from an insect, Manduca sexta: A bacteria-inducible protein similar to Drosophila easter. Proceedings of the National Academy of Sciences of the United States of America 1998, 95:12220-12225.

29. Jiang $H$, Wang $Y, Y u X Q$, Kanost MR: Prophenoloxidase-activating proteinase-2 from hemolymph of Manduca sexta: a bacteria-inducible serine proteinase containing two clip domains. Journal of Biological Chemistry 2003, 278:3552-3561.

30. De Gregorio E, Spellman PT, Rubin GM, Lemaitre B: Genome-wide analysis of the Drosophila immune response by using oligonucleotide microarrays. Proceedings of the National Academy of Sciences 2001, 98:12590-12595

31. Gorman MJ, Andreeva OV, Paskewitz SM: Molecular characterization of five serine protease genes cloned from Anopheles gambiae hemolymph. Insect Biochemistry and Molecular Biology 2000, 30:35-46.

32. Bao $Y$, Yamano $Y$, Morishima I: $\beta-1,3-$-Glucan inducible expression of prophenoloxidase-activating proteinase from eri-silkworm, Samia cynthia ricini. Comparative Biochemistry and Physiology - B Biochemistry and Molecular Biology 2007, 147:45-48.

33. Liu H, Jiravanichpaisal P, Cerenius L, Lee BL, Söderhäll I, Söderhäll K: Phenoloxidase is an important component of the defense against Aeromonas hydrophila infection in a Crustacean, Pacifastacus leniusculus. Journal of Biological Chemistry 2007, 282:33593-33598.

34. Martin GG, Poole D, Poole C, Hose JE, Arias M, Reynolds L, McKrell N, Whang A: Clearance of bacteria injected into the hemolymph of the penaeid shrimp, Sicyonia ingentis. Journal of Invertebrate Pathology 1993, 62:308-315. 
35. Tamura K, Dudley J, Nei M, Kumar S: MEGA4: Molecular Evolutionary Genetics Analysis (MEGA) software version 4.0. Mol Biol Evol 2007, 24:1596-9.

36. Nicholas KB, Nicholas HBJ: GeneDoc: a tool for editing and annotating multiple sequence alignments. Distributed by the author 1997 [http://www. psc.edu/biomed/genedoc].

doi:10.1186/1471-2172-12-70

Cite this article as: Jang et al: Selectively enhanced expression of prophenoloxidase activating enzyme 1 (PPAE1) at a bacteria clearance site in the white shrimp, Litopenaeus vannamei. BMC Immunology 2011 $12: 70$.

Submit your next manuscript to BioMed Central and take full advantage of:

- Convenient online submission

- Thorough peer review

- No space constraints or color figure charges

- Immediate publication on acceptance

- Inclusion in PubMed, CAS, Scopus and Google Scholar

- Research which is freely available for redistribution 